Chinese Journal of Organic Chemistry

\title{
三氟甲氧基化反应研究进展
}

\author{
王 \\ (南开大学化学学院 元素有机化学国家重点实验室 天津 300071)
}

\begin{abstract}
摘要 近年来, 有机氟化学领域迅猛发展, 氟化及含氟官能团化反应受到有机化学家的高度关注. 含氟官能团具有特 殊的理化性质, 将其引入药物分子会提高药物分子的生物活性. 其中三氟甲氧基具有较强的吸电子性和高亲脂性, 含 有三氟甲氧基的化合物在医药和农药等领域发挥着重要的作用. 近年, 一些富有创新性的策略被用于合成含有三氟甲 氧基的化合物. 重点论述了我们研究小组在三氟甲氧基化反应领域的研究成果, 并讨论了三氟甲氧基化反应所面临的 一些挑战.
\end{abstract}

关键词 三氟甲氧基化; 苯磺酸三氟甲酯(TFMS); $(E)-O$-三氟甲基苯甲醛肜(TFBO)

\section{Recent Advances in Trifluoromethoxylation Reactions}

\author{
Wang, Feng Tang, Pingping* \\ (State Key Laboratory of Elemento-organic Chemistry, College of Chemistry, Nankai University, Tianjin 300071)
}

\begin{abstract}
In recent years, the field of organic fluorine chemistry has developed rapidly. Fluorination and fluorine-containing functionalization reactions have attracted extensive attention of organic chemists due to their special physical and chemical properties. The introduction of fluorine-containing group into drug molecules can improve the biological activity of drug molecules. Trifluoromethoxy group has strong electron absorption and high lipophilicity, compounds containing trifluoromethoxy play an important role in the fields of medicine, pesticides and materials. In recent years, some innovative strategies have been used to synthesize compounds containing trifluoromethoxy group. This account mainly focuses on the research of trifluoromethoxy reaction in our group, and some challenges faced by trifluoromethoxy reaction.
\end{abstract}

Keywords trifluoromethoxylation; trifluoromethyl aryl sulfonate (TFMS); (E)-O-trifluoromethyl-benzaldoximes (TFBO)

自从 20 世纪中期, 含氟化合物在材料、农药、医药 和军工等领域得到广泛应用 ${ }^{[1]}$. 特别是在生命科学领域, 具有特殊性质的含氟化合物受到更多关注 ${ }^{[1 \mathrm{~g}, 2]}$. 在过去 几年中, 含有与杂原子连接的 $\mathrm{CF}_{3}$ 基团的化合物也备受 关注, 其中, 含有三氟甲氧基基团 $\left(\mathrm{OCF}_{3}\right)$ 的化合物因其 独特的物理化学性质而具有很高的应用价值. 三氟甲氧 基基团具有良好的代谢稳定性, 合适的亲脂性参数 (Hansch 参数: $\mathrm{pr}=1.04)^{[3]}$ 和特定的电子特性 (Hammett 常数: $\mathrm{sp}=0.35, \mathrm{sm}=0.38$, Swain-Lupton 常数: $F=0.39$, $R \leqslant 0.04)^{[4]}$.

值得一提的是, 含有三氟甲氧基的芳烃具有高度特 异性的结构特征 ${ }^{[5]}$, 部分含有三氟甲氧基的分子可用作 药物或农用化学品(图 1). 例如, 利鲁唑可用于治疗肌萎
缩侧索硬化症(卢伽雷氏病或夏科病), 氟酩磺隆则是防 除小麦田禾本科杂草和一些重要阔叶杂草的除草剂 ${ }^{[6]}$.

然而，尽管受到很大的关注，但只有非常有限的方 法能合成含三氟甲氧基的化合物. 传统的方法基本上是 通过氟负离子的取代反应分步构建三氟甲氧基 ${ }^{[7]}$, 这种 方法非常适合制备简单的大宗氟化工原料. 但是这些方 法经常需要苛刻的反应条件和具有腐蚀性的试剂(例如 $\mathrm{HF})$. 而对于药物研发和精细化学品的制备来说, 利用 温和条件构建三氟甲氧基的策略则更为适用. 过去几年 中, 随着有机氟化学的快速发展, 更简便、更实用的新 型合成策略已经出现 ${ }^{[8]}$, 例如羟基与三氟甲基化试剂作 用构建 $\mathrm{OCF}_{3}$ 键的策略 ${ }^{[9]}$ 以及直接引入三氟甲氧基的策 略, 这主要得益于新试剂的开发.

\footnotetext{
* Corresponding author. E-mail: ptang@nankai.edu.cn

Received March 20, 2020; revised April 24, 2020; published online April 30, 2020.

Project supported by the National Key Research and Development Program of China (No. 2016YFA0602900), the National Natural Science Foundation of China (Nos. 21672110, 21925105) and the Natural Science Foundation of Tianjin City (No. 18JCJQJC47000).

国家重点研发计划(No. 2016YFA0602900)、国家自然科学基金(Nos. 21672110, 21925105)及天津市自然科学基金(No. 18JCJQJC47000)资助项目.
} 
<smiles>CC1(C)Oc2ccc(OC(F)(F)F)cc2[C@H](N2Cc3ccccc3C2=O)[C@H]1O</smiles>

利鲁唑(Riluzole)® 肌萎缩侧索硬化症治疗<smiles>O=C(NC(=O)c1ccccc1Cl)Nc1ccc(OC(F)(F)F)cc1</smiles>

杀铃脲(Triflumuron) 杀虫剂

\section{图 1 含有三氟甲氧基的药物和农药分子}

Figure 1 Drugs and pesticide containing trifluoromethoxy group

三氟甲氧基化试剂可以分为自由基型和亲核型试 剂. 1948 年, 自由基型试剂 $\mathrm{CF}_{3} \mathrm{OF}(\mathrm{FTM})$ 问世 $^{[10]}$, 但该 试剂是剧毒气体, 成本较高, 没有得到广泛的应用. 2012 年, Tortelli 课题组 ${ }^{[11]}$ 使用该试剂实现了芳环 $\mathrm{C}\left(\mathrm{sp}^{2}\right)-\mathrm{H}$ 键的直接三氟甲氧基化. 2018 年, Ngai 课题 组 ${ }^{[12]}$ 和 Togni 课题组 ${ }^{[13]}$ 分别发展了 “ $\mathrm{NOCF}_{3}$ ” 型的自由 基型试剂, 在光照条件下产生三氟甲氧基自由基, 进而 实现芳环 $\mathrm{C}\left(\mathrm{sp}^{2}\right)-\mathrm{H}$ 键的直接三氟甲氧基化.

亲核型试剂的研究则更加深入. 20 世纪中期, 三氟 甲磺酸三氟甲酯(TFMT)的制备方法被报道 ${ }^{[14]}$, 但该试 剂沸点低 $\left(19{ }^{\circ} \mathrm{C}\right)$, 挥发性高, 制备提纯难度较大. 2008 年, 化学家发展了 TFMT 原位生成三氟甲氧基盐的策 略 ${ }^{[15]}$. 后来, 化学家利用分离的三氟甲氧基盐, 如 $\mathrm{AgOCF}_{3}{ }^{[15 \mathrm{a}, 16]}, \mathrm{CsOCF}_{3}{ }^{[17]}, \mathrm{TAS} \cdot \mathrm{OCF}_{3}$ [三(二甲氨基)锍三 氟甲醇盐 $]^{[18],} \mathrm{R}_{4} \mathrm{NOCF}_{3}{ }^{[15 \mathrm{a}, 19]}$ 等实现了多种三氟甲氧基 化反应. Langlois 课题组 ${ }^{[20]}$ 利用 2,4-二硝基三氟甲氧基 苯(DNTFB)成功实现了脱卤素直接三氟甲氧基化反应. 2017 年, 我们课题组 ${ }^{[21]}$ 发展了新型的三氟甲氧基化试 剂一一苯磺酸三氟甲酯(TFMS), 并实现了一系列不同 底物的直接三氟甲氧基化反应. 2018 年, 胡金波课题组 ${ }^{[22]}$ 发展了类似的三氟甲氧基化试剂——苯甲酸三氟甲 酯(TFBz), 并顺利地实现了苯炔的溴代三氟甲氧基化反 应. 2020 年, 我们课题组利用新发展的 $(E)-O$-三氟甲基 苯甲醛肟(TFBO), 顺利实现了脱卤三氟甲氧基化反应 [23]. 本文将重点介绍本课题组最近几年开发的新方法.

\section{1 双键不对称溴代-三氟甲氧基化反应研究}

2017 年, 我们课题组 ${ }^{[21 a]}$ 发展了一种新型三氟甲氧 基化试剂芳基磺酸三氟甲酯(TFMS). 该试剂的合成方 法简便，易于操作，通过芳基磺酸与 Togni's 试剂反应,
可以以较高产率得到 TFMS (Scheme 1).

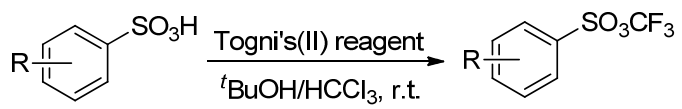

图式 1 TFMS 的制备方法

Scheme 1 Preparation of TFMS

随后我们 ${ }^{[21 a]}$ 对该试剂的反应性进行了探索，并发 现该试剂可以在氟负离子的作用下释放三氟甲氧基负 离子. 利用此特性, 在银盐的催化下首次实现了烯烃的 不对称溴代一三氟甲氧基双官能团化反应(Scheme 2). 该反应条件温和，底物适用性好，苯乙烯及链状烯烃均 能很好地参与反应; 同时, 各种吸电子和供电子官能团 在该反应条件下均有很好的容忍度. 该反应可以实现克 级规模放大; 该反应也适用于复杂天然产物及其衍生物 的后期官能团化. 此外, 对于苯乙烯类化合物，能以较 高的对映选择性得到 $(S)$ 构型为主的产物, 为后续的不 对称三氟甲氧基化反应研究提供了思路.
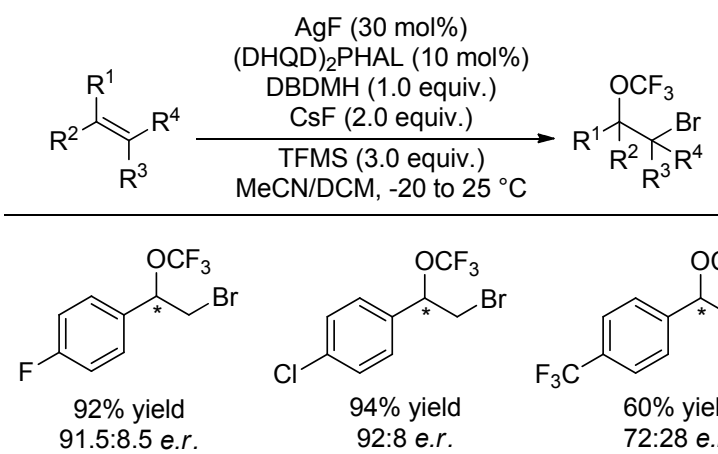<smiles></smiles><smiles>N#Cc1ccc(C(CBr)OC(F)(F)F)cc1</smiles><smiles>FC(F)(F)OC(CBr)c1ccc(-c2ccccc2)cc1</smiles><smiles>FC(F)(F)OC(CBr)c1ccccc1</smiles>

$60 \%$ yield $72: 28$ e.r.

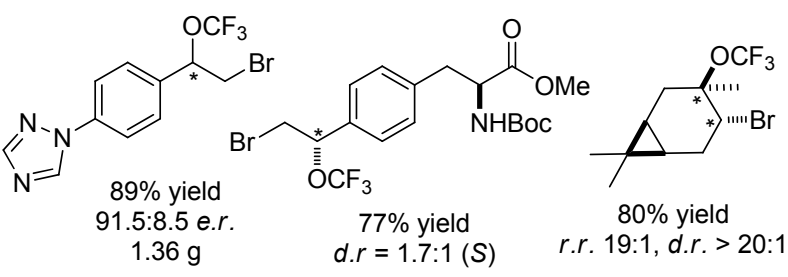

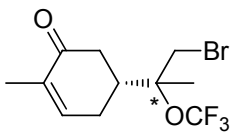

80 yield

r.r. $=14: 1$, d.r. $=3: 1$<smiles>CC(C)(Br)C(C)(C)OC(F)(F)F</smiles>

$39 \%$ yield

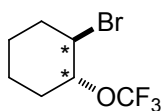

88 yield
图式 2 银催化烯烃不对称溴代一三氟甲氧基化反应底物扩展 Scheme 2 Substrates scope for asymmetric silver-catalyzed intermolecular bromotrifluoromethoxylation of simple alkenes

接下来对反应机理进行了研究: 将 TFMS 试剂与 $\mathrm{CsF}$ 混合, 通过 ${ }^{19} \mathrm{~F} \mathrm{NMR}$ 可以检测到 $\mathrm{CsOCF}_{3}$ 物种 $(\delta$ 
-21)和芳基磺酰氟的生成，如果往反应体系再加入 1.0 equiv. $\mathrm{AgF}, \mathrm{CsOCF}_{3}(\delta-21)$ 的氟峰能快速地转变成 $\mathrm{AgOCF}_{3}(\delta-25)$. 控制实验也表明, TFMS 试剂与 $\mathrm{AgF}$ 反应也能直接生成 $\mathrm{AgOCF}_{3}$, 可见反应体系中能原位生 成 $\mathrm{AgOCF}_{3}$ 物种(Scheme 3).
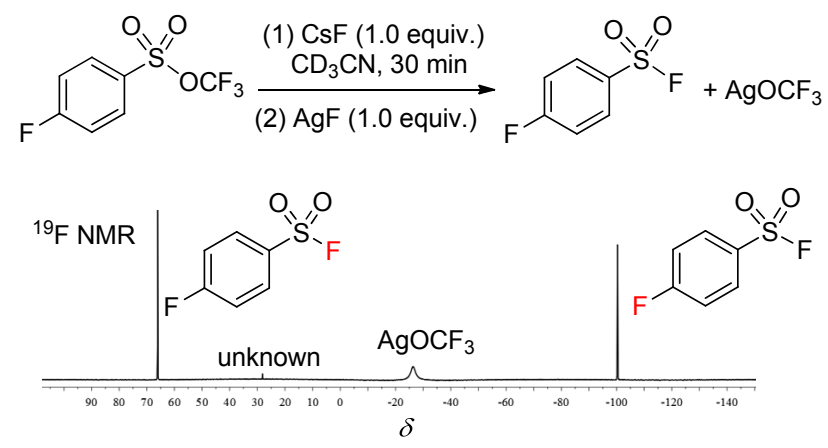

图式 3 银催化烯烃不对称溴代-三氟甲氧基化反应机理研究 Scheme 3 Mechanism research for asymmetric silver-catalyzed intermolecular bromotrifluoromethoxylation of simple alkenes

基于上述实验证据和反应的立体化学, 提出一种可 能的反应机理(图 2): 首先烯烃底物通过 $\pi-\pi$ 重叠进入奎 宁催化剂的手性空间, 催化剂上的三级胺活化 1,3-二澳5,5-二甲基海因(DBDMH), 使得溴与烯烃的双键发生作 用, 奎宁的氮原子和 $\mathrm{AgOCF}_{3}$ 有络合作用, 使得三氟甲 氧基负离子按一定的方位进攻烯键，从而生成了具有立 体选择性的目标产物.

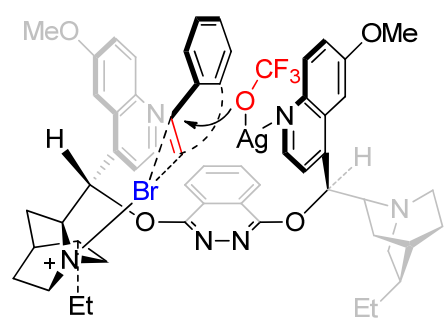

图 2 银催化烯烃不对称溴代一三氟甲氧基化反应机理

Figure 2 Insights into the reaction mechanism of asymmetric silver-catalyzed intermolecular bromotrifluoromethoxylation of alkenes

\section{2 烷基醇脱氧一三氟甲氧基化反应研究}

通过对 TFMS 反应性的考察, 发现三氟甲氧基负离 子具有一定的亲核性. 同时也存在着向二氟光气分解的 平衡反应(Scheme 4a). 因此猜想, 如果利用二氟光气与 底物上的羟基作用, 原位形成比羟基更容易离去的氟甲 酰酯基团, 再利用三氟甲氧基负离子的亲核性, 就能实 现醇类底物的脱氧三氟甲氧基化反应(Scheme 4b).

我们 ${ }^{[21 b]}$ 通过不断考察, 发现以醇作为底物, 在四 甲基溴化铵为添加剂的条件下, 以不错的产率得到了相 (a)<smiles></smiles>

(b)

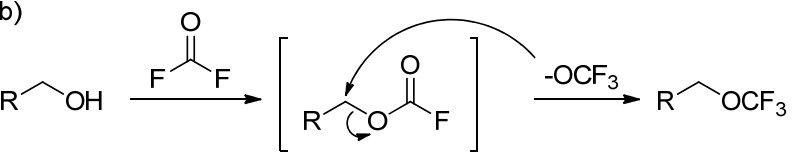

图式 4 烷基醇脱氧一三氟甲氧基化反应机理

Scheme 4 Mechanism for direct dehydroxytrifluoromethoxylation of alcohols

应的目标产物. 对反应底物进行了拓展, 发现一级醇和 二级醇均有不错的产率. 对于活性较高的茮醇类底物, 该反应也具有良好的适用性. 底物分子中如果同时存在 一级醇与二级醇, 反应优先发生在一级醇的位置. 该反 应同时适用于复杂分子的后期修饰，均能以中等左右收 率得到相应的目标产物(Scheme 5).

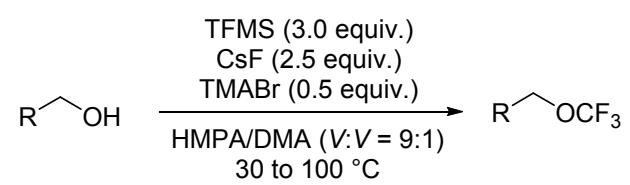<smiles>CC(CCc1ccccc1)OC(C)CCc1ccccc1</smiles>

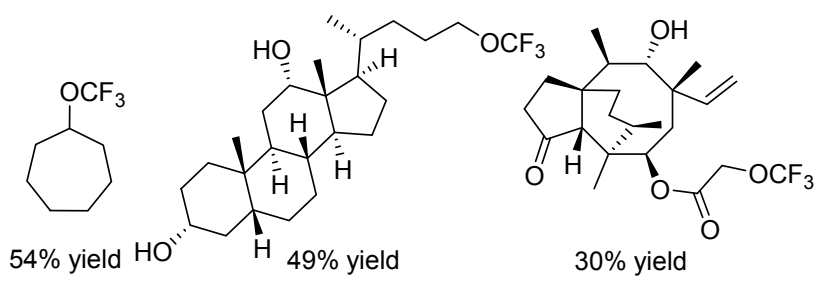

图式 5 烷基醇脱氧一三氟甲氧基化反应底物扩展 Scheme 5 Substrate scope for dehydroxytrifluoromethoxylation of alcohols

\section{3 钴催化的环氧开环-三氟甲氧基化反应研究}

2018 年, 我们课题组 ${ }^{[21 \mathrm{c}]}$ 以环氧化合物为底物, 在 钴催化剂的作用下, 顺利开环得到了三氟甲氧基化产 物. 利用钴催化剂与氧原子配位, 从而增强氧原子的离 去能力，进而利用三氟甲氧基负离子的亲核性，实现环 氧开环. 该反应的产率普遍较高, 区域选择性较好, 对 于两边位阻有差异的底物, 三氟甲氧基负离子倾向于从 位阻小的一端进攻. 然而当使用 2-苯基环氧乙烷为底物 时, 区域选择性较差, 这是底物的空间位阻与电性共同 影响所致. 当底物上含有两个不同位阻的环氧官能团 时, 该反应优先在位阻较小的一端进行(Scheme 6). 

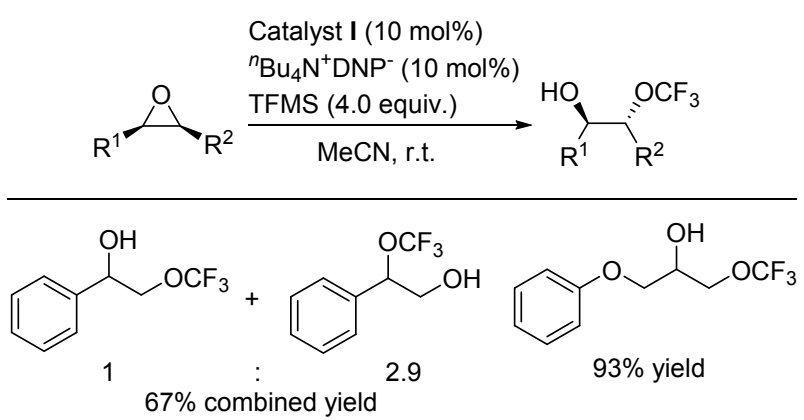<smiles>CCOCC(O)CCc1ccccc1</smiles>

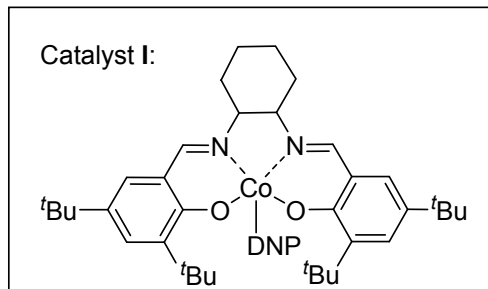

$\mathrm{DNP}=$ 2,4-dinitrophenoxy

图式 6 环氧开环-三氟甲氧基化反应底物扩展 Scheme 6 Substrate scope for ring-opening reaction of epoxides

\section{4 光催化双键叠氮-三氟甲氧基化反应研究}

有别于传统的亲核取代反应，我们同样开发了氧化 底物形成碳正离子的策略, 充分利用三氟甲氧基负离子 的亲核性，顺利实现了多种三氟甲氧基化反应. 2018 年， 我们课题组 ${ }^{[21 \mathrm{~d}]}$ 利用 TFMS 首次实现了光催化的苯乙烯 叠氮一三氟甲氧基双官能团化反应. 该反应条件温和, 多种供电子基团和吸电子基团在该条件下均有较好的 容忍度. 对不同电性的苯乙烯进行了考察, 发现含有供 电子基团的苯乙烯为底物时产率较高, 含有吸电子基团 的苯乙烯为底物时产率较低, 一定程度上说明该反应可 能经历了形成苄基碳正离子的机理. 该反应也可以用于 复杂分子的后期修饰(Scheme 7).

通过机理实验的佐证, 提出如下可能的反应机理: 首先在可见光照射下, 光敏剂 $\left[R u(b p y)_{3}\right]^{2+}$ 受到激发, 随 后被叠氮试剂氧化淬灭生成 $\left[\mathrm{Ru}(\mathrm{bpy})_{3}\right]^{3+}$ 物种和叠氮自 由基, 接着叠氮自由基对底物双键进行加成生成茮位自 由基, 芐位自由基又被 $\left[\mathrm{Ru}(\mathrm{bpy})_{3}\right]^{3+}$ 物种氧化生成碳正 离子中间体, 进而被体系中原位形成的 $\mathrm{AgOCF}_{3}$ 物种捕 获得到目标产物(Scheme 8).

\section{5 芒位 C一H 键的三氟甲氧基化反应研究}

上述实验中我们发现在氧化剂存在的情况下，茮基

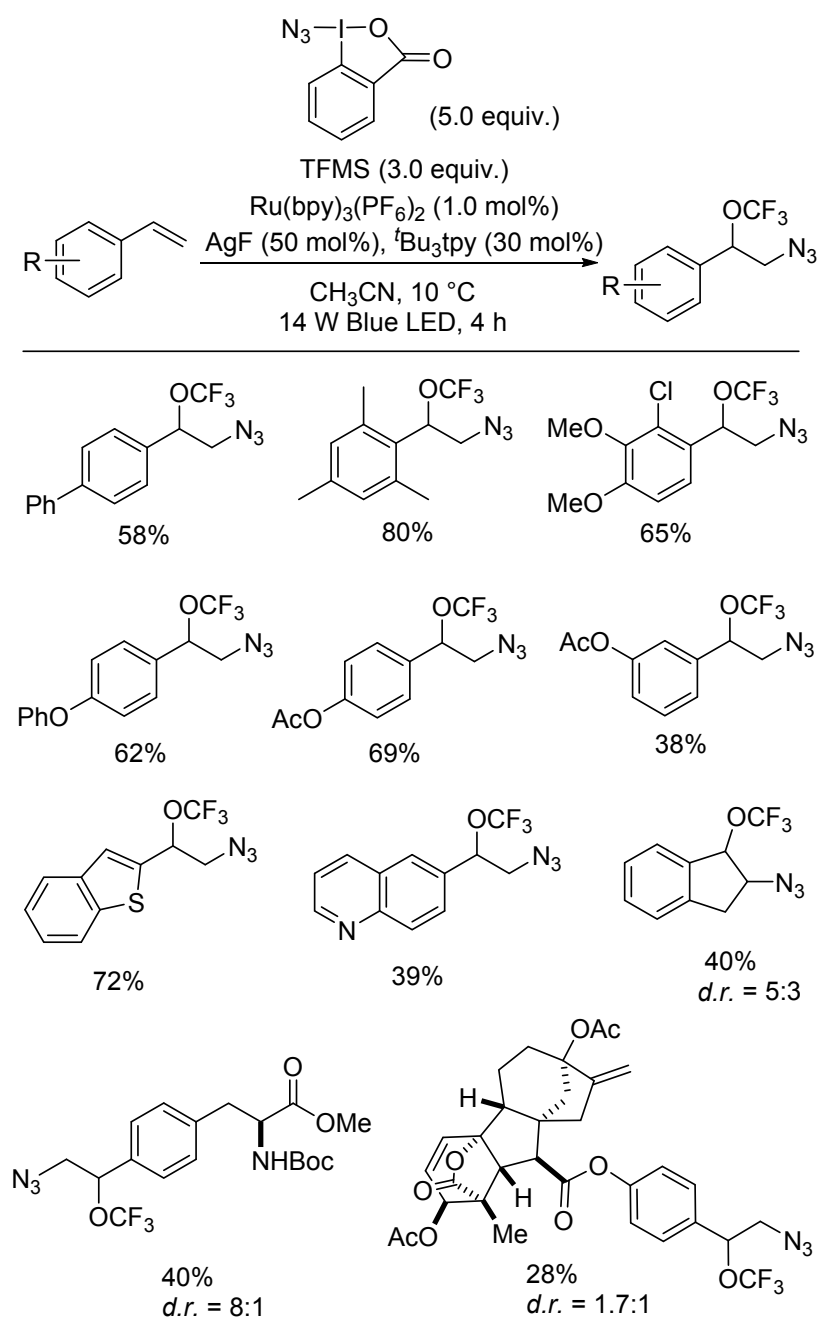

图式 7 烯烃的叠氮-三氟甲氧基化反应底物扩展 Scheme 7 Substrate scope for azidotrifluoromethoxylation of styrenes

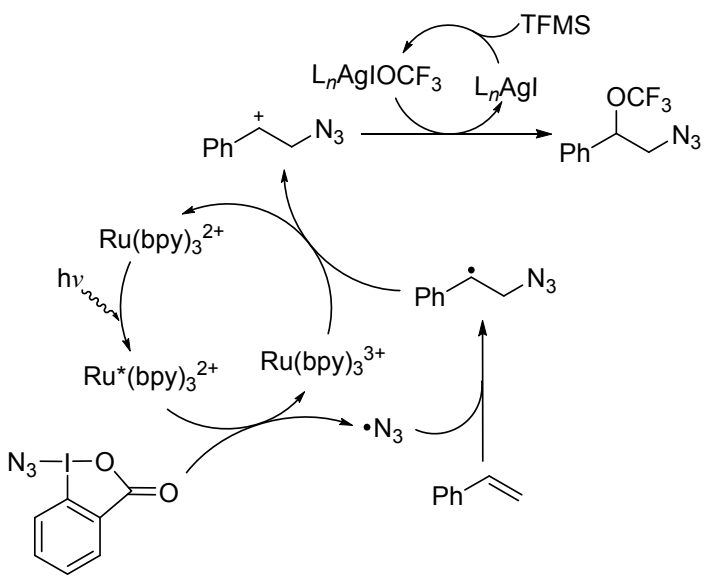

图式 8 烯烃的叠氮-三氟甲氧基化反应机理 Scheme 8 Mechanism of azidotrifluoromethoxylation of styrenes

自由基可以被进一步氧化为碳正离子，进而与三氟甲氧 基负离子结合形成产物. 结合本组早期的工作 ${ }^{[24]}$ 猜想, 
在合适的氧化剂作用下, 通过对芐位 $\mathrm{C}-\mathrm{H}$ 键的直接活 化是否可以经历类似上述反应历程, 形成芐位含有三氟 甲氧基的化合物. 通过不断考察, 2018 年, 我们课题 组 ${ }^{[21 e]}$ 实现了银催化/促进的苄位 $\mathrm{C}\left(\mathrm{sp}^{3}\right)-\mathrm{H}$ 键直接三氟 甲氧基化反应. 该反应条件均较为温和, 但对于不同电 性的底物需要使用不同的条件: 连有供电子基的底物使 用过硫酸钾为氧化剂, 产率普遍较高; 对于连有吸电子 基的底物则需要 $\mathrm{AgF}_{2}$ 为氧化剂参与反应的转化. 该反 应有较好的底物适用范围, 对于一级碳和二级碳底物均 有一定的适用性, 对一些复杂小分子的后期修饰也同样 适用. 底物分子中同时存在一级碳和二级碳, 反应在二 级碳上发生反应的速率快于一级碳. 而当分子中同时存 在一级碳和三级碳, 反应主要发生在一级碳上(表 1). 值 得注意的是, 如果使用 1-氯甲基-4-氟-1,4-重氮化双环 [2.2.2]辛烷二 (三氟甲磺酸盐) (F-TEDA-Otf)作为氧化剂, 对于一些连有强供电子基的底物, 该反应还能得到芐位 同时氟化和三氟甲氧基化的产物(Scheme 9).

表 1 芐位 $\mathrm{C}-\mathrm{H}$ 键的三氟甲氧基化反应底物扩展 Table 1 Substrate scope of the benzylic C-H trifluoromethoxylation

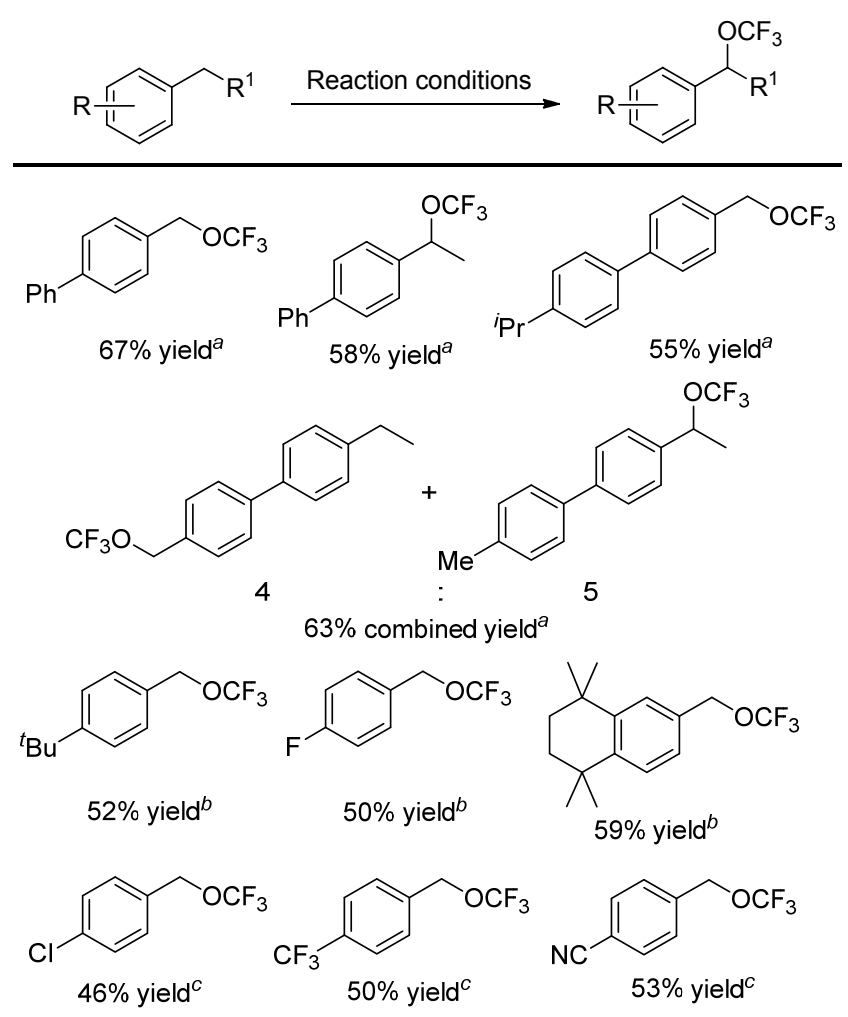

${ }^{a}$ Reaction conditions: $\operatorname{AgOTf}(30 \mathrm{~mol} \%), \mathrm{K}_{2} \mathrm{~S}_{2} \mathrm{O}_{8}$ (3.0 equiv.), $\mathrm{CsF}$ (4.0 equiv.), TFMS (5.0 equiv.), 1,10-phenanthroline-5,6-dione ( $5 \mathrm{~mol} \%$ ), DMC, $50{ }^{\circ} \mathrm{C} .{ }^{b}$ Reaction conditions: AgOTf (30 mol\%), F-TEDA-OTf (3.0 equiv.), CsF (4.0 equiv.), TFMS (3.0 equiv.), 1,10-phenanthroline-5,6-dione ( $5 \mathrm{~mol} \%$ ), 4,7-diphenyl-1,10-phenanthroline (5 mol\%), DMC, $25{ }^{\circ} \mathrm{C} .{ }^{c}$ Reaction conditions: $\mathrm{AgF}_{2}$ (6.0 equiv.), 4,4'-dimethyoxy-2,2'-bipyridine (10 mol\%), di(2pyridinyl)-methanone (10 mol\%), TFMS (4.0 equiv.), $\mathrm{MeCN}, 25{ }^{\circ} \mathrm{C}$.

通过机理实验和计算化学的佐证提出了可能的反

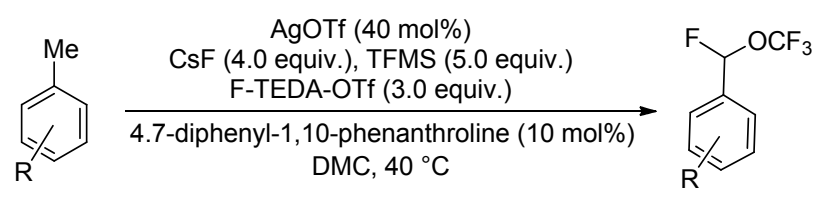

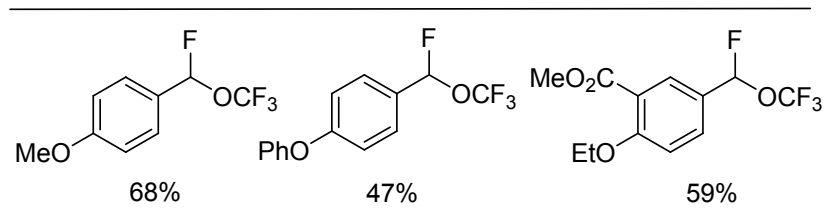<smiles>CCCCCOc1ccc(C(F)OCCCCCC(C)(C)C(F)(F)F)cc1</smiles>

图式 9 苄位 $\mathrm{C}-\mathrm{H}$ 键的氟化三氟甲氧基化反应底物范围 Scheme 9 Substrate scope of the benzylic C-H trifluoromethoxylation and fluorination

应机理(Scheme 10). 首先 AgOTf 在体系中与氟离子交 换得到 $\mathrm{AgF}$, 经过硫酸钾氧化形成 $\mathrm{Ag}(\mathrm{II})$ 物种, 之后与 TFMS 作用生成 F-Ag ${ }^{\mathrm{II}}-\mathrm{OCF}_{3}$ 的活性中间体. 底物被过 硫酸钾氧化产生茮基自由基. 茮位自由基进而被 $\mathrm{Ag}^{\mathrm{II}}$ 物 种氧化为碳正离子，接着碳正离子中间体与体系中原位 形成的 $\mathrm{AgOCF}_{3}$ 物种作用生成目标产物 (path A). 另外通 过计算表明, 茮位自由基同样能够从 $\mathrm{F}-\mathrm{Ag}^{\mathrm{II}}-\mathrm{OCF}_{3}$ 物种 直接篗取 $\mathrm{OCF}_{3}$ 生成目标产物和 $\mathrm{AgF}$, 从而形成产物 (path B).

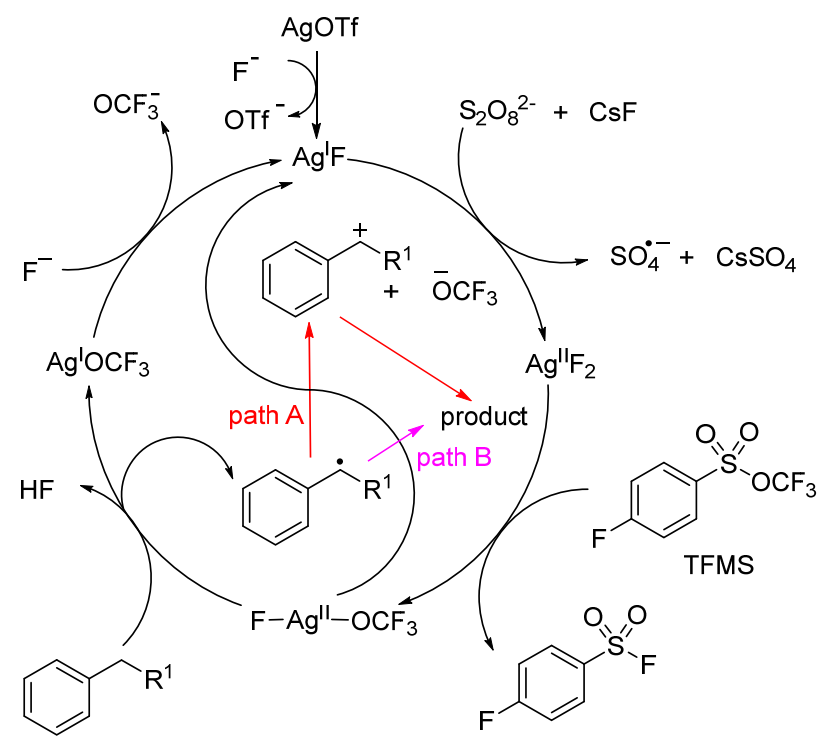

图式 10 苄位 $\mathrm{C}-\mathrm{H}$ 键的三氟甲氧基化反应机理 Scheme 10 Mechanism of the benzylic C-H trifluoromethoxylation reaction

\section{6 烷基硅化合物的三氟甲氧基化反应研究}

上述两例转化中均涉及茮基自由基的形成, 在氧化 剂的条件下形成苠位连有三氟甲氧基的化合物. 我们猜 
想, 是否其他类型碳自由基可以在氧化剂的条件下也能 实现上述转化. 结合本组早期烷基硅化合物的氟化反 应 $^{[25]}$ 经验：烷基硅在氟负离子及氧化剂的作用下可以 形成碳自由基. 我们 ${ }^{[21 \mathrm{f}]}$ 尝试并实现了首例银促进的烷 基硅化合物的三氟甲氧基化反应. 该反应条件温和，适 用于一级碳的硅烷底物, 含有吸电子基或供电子基的底 物均能得到良好的产率. 而对于二级碳的硅烷底物反应 产率则明显降低. 该反应也适用于复杂分子的后期修饰 (Scheme 11). 该反应也存在不足: AgF 及 TFMS 用量较 多, 底物类型较为单一. 自由基验证实验表明, 该反应 可能经历了碳自由基的历程.

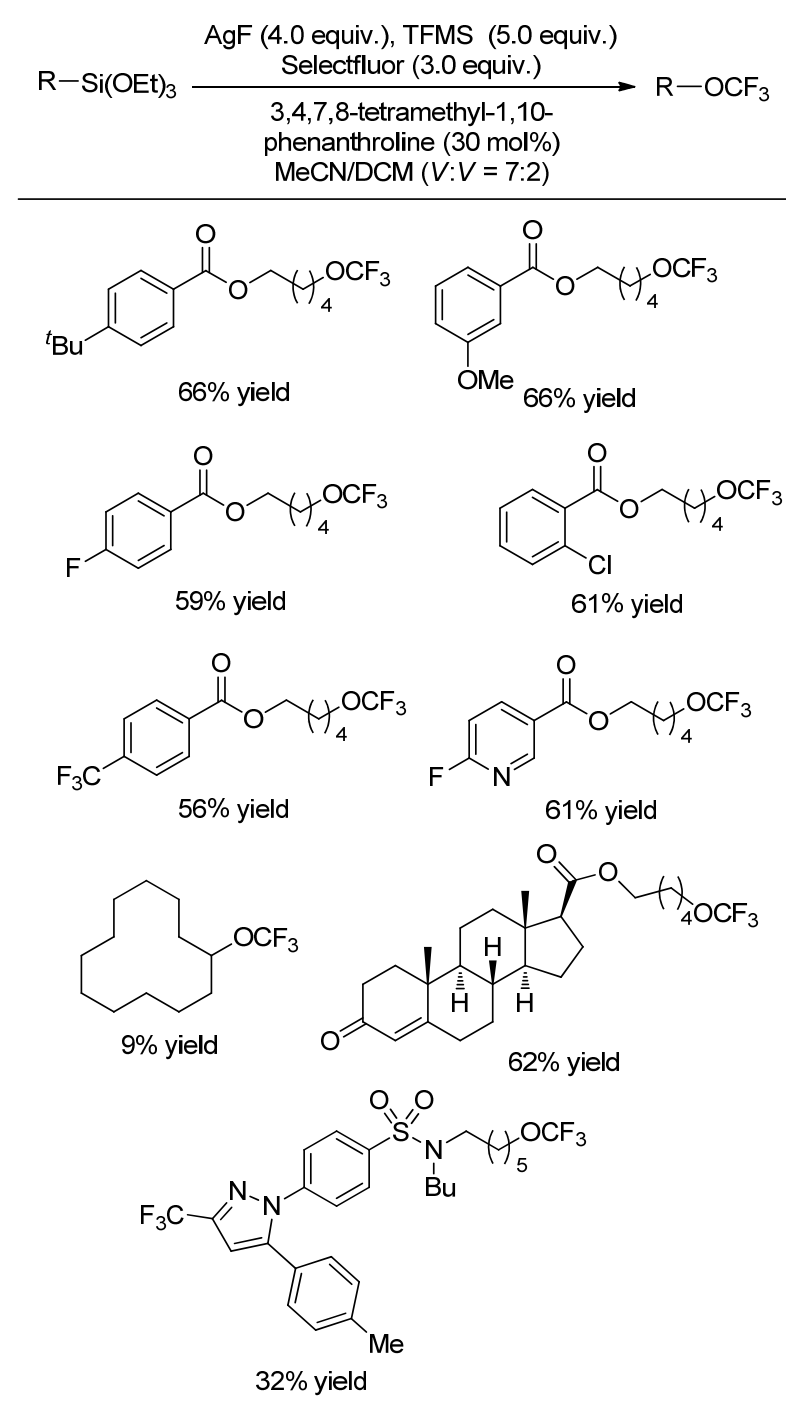

图式 11 银促进的烷基硅的三氟甲氧基化反应底物扩展 Scheme 11 Substrate scope for silver-mediated oxidative trifluoromethoxylation of alkylsilanes

\section{7 光催化铜促进的芳基重氮盐的三氟甲氧基化 反应研究}

在构建 $\mathrm{C}\left(\mathrm{sp}^{3}\right)-\mathrm{OCF}_{3}$ 键的同时, 我们也对 $\mathrm{C}\left(\mathrm{sp}^{2}\right)-$
$\mathrm{OCF}_{3}$ 键的构筑方法进行了探究. 考察了传统构筑 $\mathrm{C}\left(\mathrm{sp}^{2}\right)-\mathrm{C}$ 和 $\mathrm{C}\left(\mathrm{sp}^{2}\right)-\mathrm{X}$ 键的前体如芳基硼酸、芳基卤代 物和芳基重氮盐等的反应活性，最终选用活性较高的芳 基重氮盐作为底物进行考察. 最终, 我们课题组 ${ }^{[21 g]}$ 实 现了通过 $\mathrm{Ru}$ 光敏剂催化、铜盐介导的芳基重氮盐的三 氟甲氧基化反应. 该反应产率普遍较高. 多种官能团如 烷基溴、芳基碘和三氟甲磺酸酯等基团在该反应条件下 可以很好地保留，然而若使用邻位连有取代基的底物， 该反应的产率会降低. 另外该反应适用复杂底物的后期 修饰(Scheme 12).

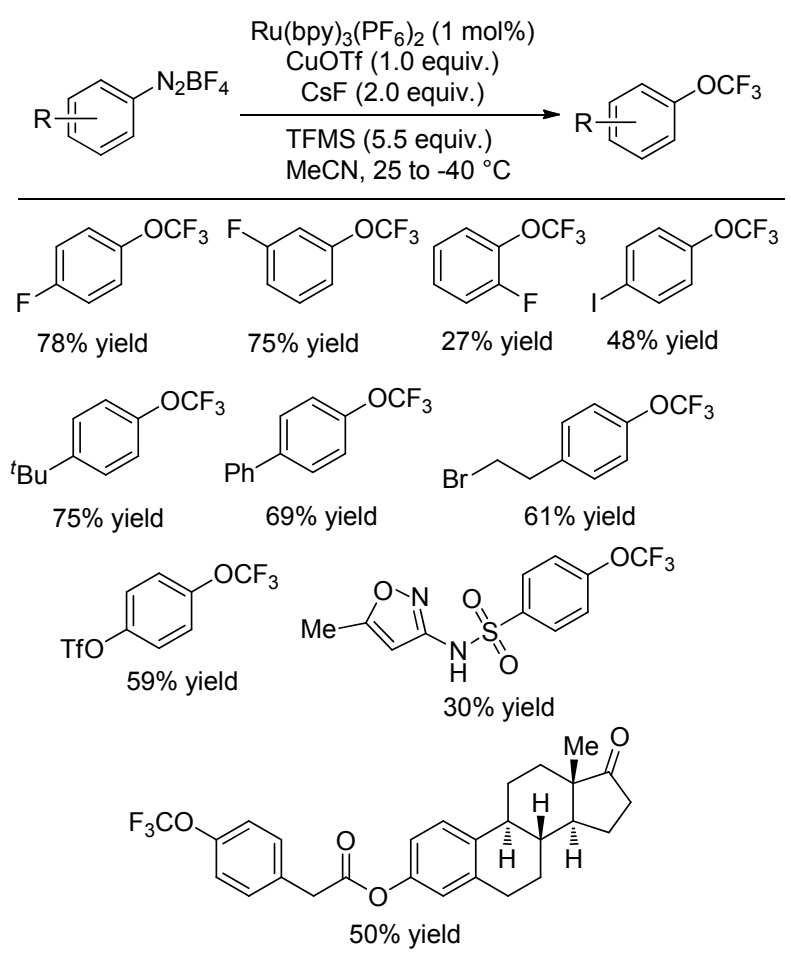

图式 12 芳基重氮盐的三氟甲氧基化反应底物扩展 Scheme 12 Substrate scope for trifluoromethoxylation of aryldiazonium salts

通过机理实验的验证提出了如下可能的反应机理: 在可见光照射下，基态光敏剂 $\left[R u(b p y)_{3}\right]^{2+}$ 形成激发态 $\left[\mathrm{Ru} *(\mathrm{bpy})_{3}\right]^{2+}$ 物种, 该物种由重氮盐氧化淬灭, 通过单 电子转移过程, $\left[\mathrm{Ru}^{*}(\mathrm{bpy})_{3}\right]^{2+}$ 被氧化为 $\left[\mathrm{Ru}(\mathrm{bpy})_{3}\right]^{3+}$, 重 氮盐则脱除氮气转变为芳基自由基. 同时, 在 $\mathrm{CsF}$ 存在 下, CuOTf 和 TFMS 原位产生 $\mathrm{Cs}\left[\mathrm{Cu}\left(\mathrm{OCF}_{3}\right)_{2}\right]$ 物种. 芳基 自由基与 $\mathrm{Cs}\left[\mathrm{Cu}\left(\mathrm{OCF}_{3}\right)_{2}\right]$ 物种作用形成 $\mathrm{Cu}$ (II)物种. 接着 $\mathrm{Cu}(\mathrm{II})$ 物种被 $\left[\mathrm{Ru}(\mathrm{bpy})_{3}\right]^{3+}$ 氧化为 $\mathrm{Cu}(\mathrm{III})$ 物种, 同时 $\left[\mathrm{Ru}(\mathrm{bpy})_{3}\right]^{3+}$ 重新被还原为 $\left[\mathrm{Ru}(\mathrm{bpy})_{3}\right]^{2+}$. 随后 $\mathrm{Cu}(\mathrm{III})$ 物 种发生还原消除得到最终产物, 并形成 $\mathrm{Cu}(\mathrm{I})$ 物种 (Scheme 13).

\section{8 烷基卤代物的亲核三氟甲氧基化反应研究}

2020 年, 我们课题组 ${ }^{[23]}$ 发展了以 $(E)-O$-三氟甲基苯 


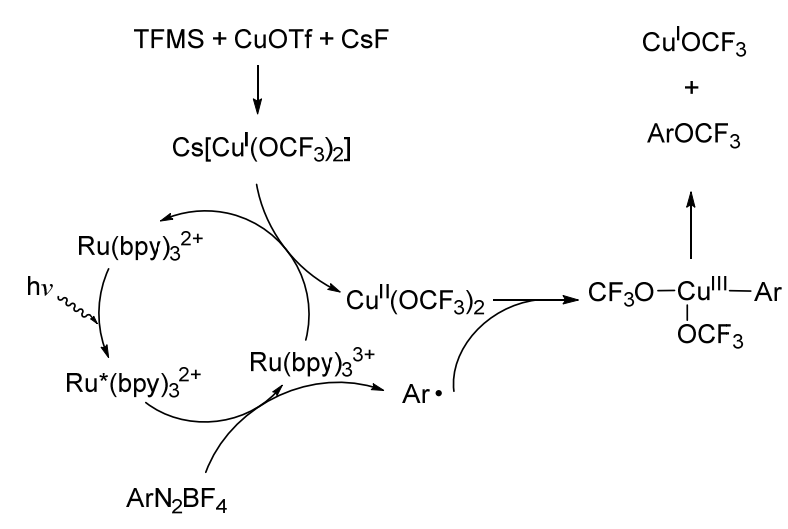

图式 13 芳基重氮盐的三氟甲氧基化反应机理

Scheme 13 Mechanism for trifluoromethoxylation of aryldiazonium salts

甲醛肜(TFBO) 作为三氟甲氧基化试剂. 通过苯甲醛与 $\mathrm{N}$-三氟甲氧基邻苯二甲酰亚胺作用, 得到了相应的产物 (Scheme 14).

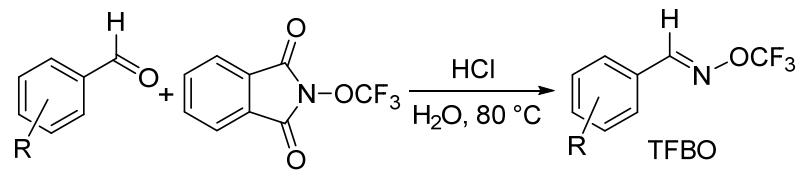

图式 14 TFBO 的制备方法

Scheme 14 Preparation of TFBO

随后考察了该试剂的反应活性, 发现该试剂可以在 碱的作用下形成三氟甲氧基负离子, 从而使得该试剂可 以用于三氟甲氧基化反应中(Scheme 15). 我们从最简 单的烷基卤代物的亲核取代反应入手, 进行了尝试并取 得了成功, 其中碱的使用对于 TFBO 的高效转化至关重 要, 在使用 $\mathrm{Cs}_{2} \mathrm{CO}_{3}$ 作碱时, 该反应以最高的产率得到相 应的产物.

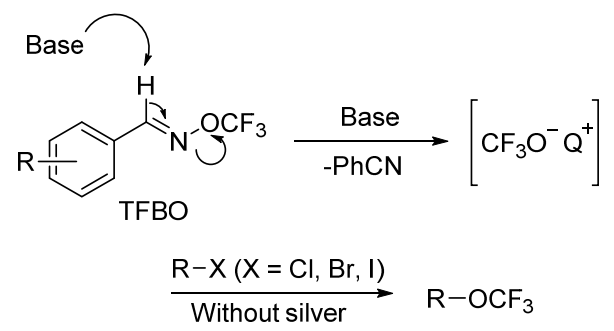

图式 15 碱促进的烷基卤代物的三氟甲氧基化反应 Scheme 15 Base-promoted trifluoromethoxylation of alkyl halides

相比之前的方法 ${ }^{[15 a, 20-22]}$, 我们开发的方法优势更为 明显. 首先, 传统的方法需要使用活化的底物, 如烯丙 基卤代物和茮基卤代物等, 且需要用到化学计量的银 盐. 而我们的方法拓展了底物范围, 适用于非活化位点 以及较为惰性的烷基氯代物的亲核三氟甲氧基化过程,
且避免使用银盐(Scheme 16).

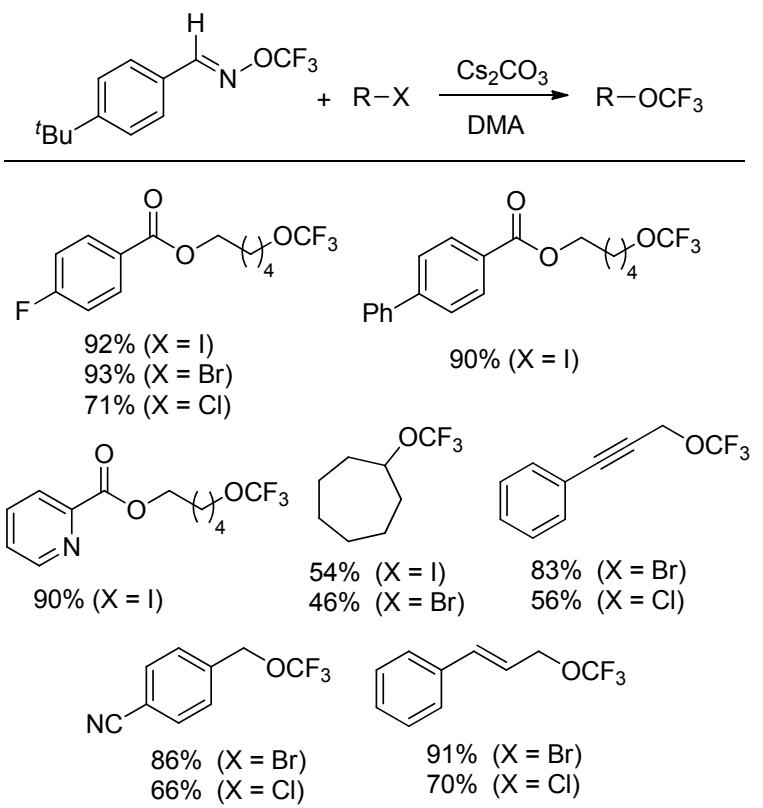

图式 16 简单烷基卤代物的三氟甲氧基化反应底物扩展 Scheme 16 Substrates scope for trifluoromethoxylation of simple alkyl halides

该反应同样适用于复杂分子的后期修饰，例如对塞 来昔布的苄位进行溴代，随后进行亲核三氟甲氧基化取 代，以 77\%的产率得到了相应的产物; 对底物分子如依 折麦布中含有醇羟基的位点进行澳代，随后进行亲核三 氟甲氧基化取代，以 $62 \%$ 的产率和 $1: 1$ 的 $d . r$. 值得到了 相应的取代产物. 说明该方法在药物分子合成等领域具 有很好的潜在应用价值(Scheme 17).

\section{9 总结与展望}

综上所述, 我们课题组基于本组发展的新型三氟甲 氧基化试剂, 发展了一系列直接三氟甲氧基化方法. TFMS 及 TFBO 试剂的应用使得三氟甲氧基化反应更为 高效，条件更为温和，操作更为简便. 可以预见，新型 三氟甲氧基化试剂将在有机化学中得到更加广泛的应 用.

然而，尽管发展了多种构建 $\mathrm{C}-\mathrm{OCF}_{3}$ 键的方法，直 接向分子中引入三氟甲氧基的策略依然面临着很多挑 战. 首先, 从 $\mathrm{C}-\mathrm{O}$ 键的类型来讲, 相比于 $\mathrm{C}\left(\mathrm{sp}^{3}\right)-$ $\mathrm{OCF}_{3}$ 键的构建方式, 构建 $\mathrm{C}\left(\mathrm{sp}^{2}\right)-\mathrm{OCF}_{3}$ 键的方法相对 较少; 构建 $\mathrm{C}(\mathrm{sp})-\mathrm{OCF}_{3}$ 键的方法也鲜有报道. 其次, 从试剂类型来讲, 亲电型三氟甲氧基化试剂也未曾被报 道. 另外, 高立体选择性地向分子中引入三氟甲氧基的 方法依然不多. 因此，探究更为高效简洁、普适性广的 三氟甲氧基化反应与开发新型的三氟甲氧基化试剂是 有机氟化学的热门领域. 

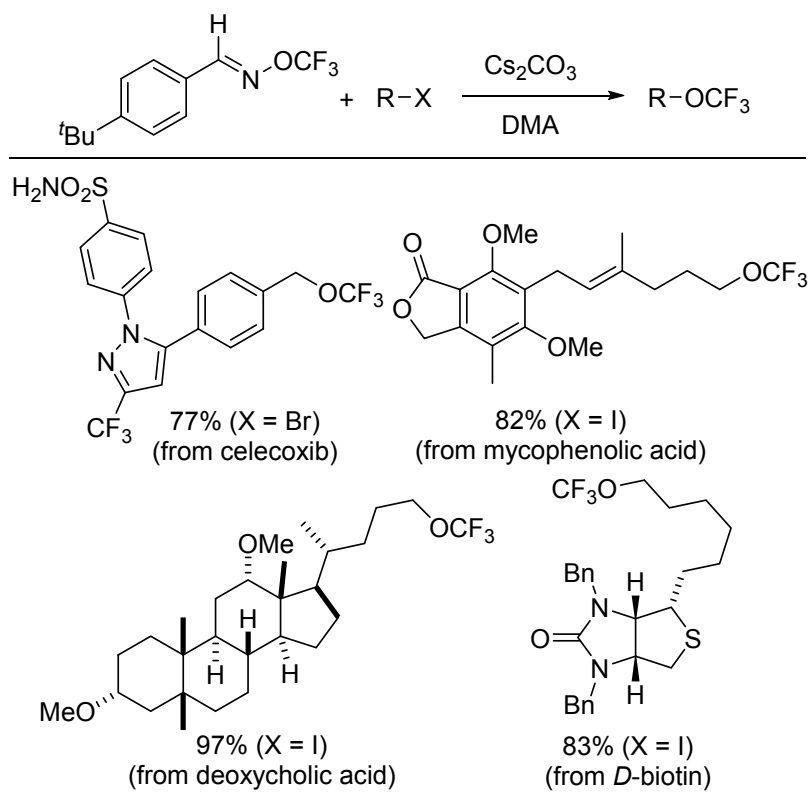

$83 \%(\mathrm{Xn}=$ (from $D$-biotin)<smiles>COC1=C(OC)C(=O)C(CCCCCCCCCCOC(F)(F)F)=C(C)C1=O</smiles>

$84 \%(X=1)$

(from idebenone)

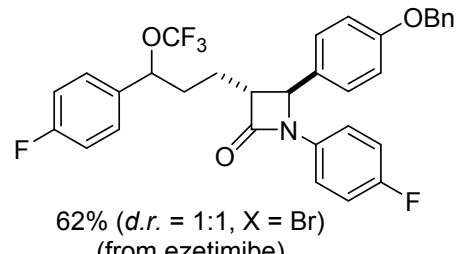

(from ezetimibe)
图式 17 复杂烷基卤代物的三氟甲氧基化反应底物扩展 Scheme 17 Substrates scope for trifluoromethoxylation of complex alkyl halides

\section{作者简介}

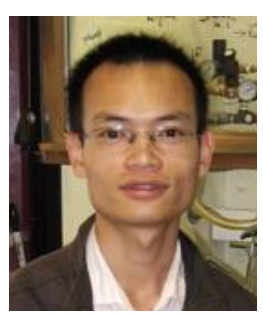

汤平平, 博士, 南开大学元素有机化学国家重点实 验室教授. 1998 2002 年就读南开大学化学系并获得学 士学位, 2002 2007 年就读于中国科学院上海有机化学 研究所并获得博士学位, 2007 2008 年于中国科学院上 海有机化学研究所担任助理研究员, 2008 2012 年于哈 佛大学化学与化学生物学系从事博士后研究. 2012 年至 今于南开大学元素有机化学国家重点实验室担任教授.

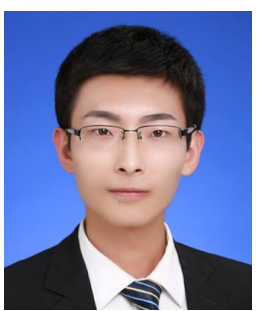

王丰, 2010～2014 年就读于天津大学化工学院并获
得学士学位, 2014 2017 年就读于南开大学化学学院并 获得硕士学位, 2017 年至今就读于南开大学化学学院, 导师为汤平平教授. 博士期间主要从事有机氟化学研 究.

\section{References}

[1] (a) Becker A. Inventory of Industrial Fluoro-biochemicals, Eyrolles, Paris, 1996.

(b) Smart, B. E. J. Fluorine Chem. 2001, 109, 3.

(c) Dunitz, J. D. ChemBioChem 2004, 5, 614.

(d) Dolbier, W. R. J. Fluorine Chem. 2005, 126, 157.

(e) Hird, M. Chem. Soc. Rev. 2007, 36, 2070

(f) Pagliaro, M.; Ciriminna R. J. Mater. Chem. 2005, 15, 4981.

(g) Wang, J.; Snchez-Rosell, M.; AceÇa, J. L.; del Pozo, C.; Sorochinsky, A. E.; Fustero, S.; Soloshonok, V. A.; Liu, H. Chem. Rev. 2014, 114, 2432

(h) Jeschke, P. ChemBioChem 2004, 5, 570.

[2] (a) Hagmann, W. K. J. Med. Chem. 2008, 51, 4359. (b) Mller, K.; Faeh, C.; Diederich, F. Science 2007, 317, 1881

[3] Leo, A.; Hansch, C.; Elkins, D. Chem. Rev. 1971, 71, 525.

[4] Hansch, C.; Leo, A.; Taft, R. W. Chem. Rev. 1991, 91, 165.

[5] (a) Kapustin, E. G.; Bzhezovsky, V. M.; Yagupolskii, L. M. J. Fluorine Chem. 2002, 113, 227.

(b) Klocker, J.; Karpfen, A.; Wolschann, P. Chem. Phys. Lett. 2003, $367,566$.

(c) Manteau, B.; Genix, P.; Brelot, L.; Vors, J. P.; Pazenok, S.; Giornal, F.; Leuenberger, C.; Leroux, F. R. Eur. J. Org. Chem. 2010, 6043.

[6] Moujalled, D.; White, A. R. CNS Drugs 2016, 30, 227.

[7] (a) Yagupol'skii, L. M. Dokl. Akad. Nauk SSSR 1955, 105, 100.

(b) Yarovenko, N. N.; Vasileva, A. S. Zh. Obshch. Khim. 1958, 28, 2502.

(c) Yagupol'skii, L. M.; Troitskaya, V. I. J. Gen. Chem. USSR 1961 31,845 .

(d) Yagupol'skii, L. M.; Orda, V. V. Zh. Obshch. Khim. 1964, 34, 1979.

(e) Manteau, B.; Genix, P.; Brelot, L.; Vors, J. P.; Pazenok, S.; Giornal, F.; Leuenberger, C.; Leroux, F. R. Eur. J. Org. Chem. 2010, 6043.

(f) Sheppard, W. A. J. Org. Chem. 1964, 29, 1.

(g) Kuroboshi, M.; Suzuki, K.; Hiyama, T. Tetrahedron Lett. 1992, 33, 4173.

(h) Kanie, K.; Tanaka, Y.; Suzuki, K.; Kuroboshi, M.; Hiyama, T. Bull. Chem. Soc. Jpn. 2000, 73, 471.

(i) Kuroboshi, M.; Hiyama, T. Adv. Synth. Catal. 2001, 343, 235.

[8] For recent reviews on trifluoromethoxylation reactions, see: (a) Besset, T.; Jubault, P.; Pannecouke, X.; Poisson, T. Org. Chem. Front. 2016, 3, 1004

(b) Tlili, A.; Toulgoat, F.; Billard, T. Angew. Chem., Int. Ed. 2016, $57,11726$.

(c) Lee, K. N.; Lee, J. W.; Ngai, M. Y. Tetrahedron 2018, 74, 7127.

(d) Lee, J. W.; Lee, K. N.; Ngai, M. Y. Angew. Chem., Int. Ed. 2019, $58,11171$.

(e) Hardy, M. A.; Chachignon, H.; Cahard, D. Asian J. Org. Chem. 2019, 8, 591

(f) Zhang, X. F.; Tang, P. P. Sci. China: Chem. 2019, 62, 525.

(g) Jiang, X. H.; Tang, P. P. Chin. J. Chem. 2020, 38, 101.

[9] (a) Umemoto, T.; Adachi, K.; Ishihara, S. J. Org. Chem. 2007, 72 , 6905.

(b) Stanek, K.; Koller, R.; Togni, A. J. Org. Chem. 2008, 73, 7678.

(c) Liang, A.; Han, S.; Liu, Z.; Wang, L.; Li, J.; Zou, D.; Wu, Y. Chem.-Eur. J. 2016, 22, 5102.

(d) Koller, R.; Huchet, Q.; Battaglia, P.; Welch, J. M.; Togni, A. Chem. Commun. 2009, 5993.

(e) Santschi, N.; Geissbhler, P.; Togni, A. J. Fluorine Chem. 2012 , 
135,83 .

(f) Matousek, V.; Pietrasiak, E.; Sigrist, L.; Czarniecki, B.; Togni, A. Eur. J. Org. Chem. 2014, 3087.

(g) Hojczyk, K. N.; Feng, P.; Zhan, C.; Ngai, M. Y. Angew. Chem., Int. Ed. 2014, 53, 14559.

(h) Chu, L.; Qing, F. L. Acc. Chem. Res. 2014, 47, 1513.

(i) Liu, J. B.; Chen, C.; Chu, L.; Chen, Z. H.; Xu, X. H.; Qing, F. L. Angew. Chem., Int. Ed. 2015, 54, 11839.

(j) Liu, J. B.; Xu, X. H.; Qing, F. L. Org. Lett. 2015, 17, 5048.

(k) Naumann, D.; Kischkewitz, J. J. Fluorine Chem. 1990, 46, 265.

(l) Hojczyk, K. N.; Feng, P.; Zhan, C.; Ngai, M. Y. Angew. Chem., Int. Ed. 2014, 53, 14559.

(m) Lee, K. N.; Lee, J. W.; Ngai, M. Y. Synlett 2016, 27, 313.

[10] Kellogg, K. B.; Cady, G. H. J. Am. Chem. Soc. 1948, 70, 3986.

[11] Venturini, F.; Navarrini, W.; Famulari, A.; Sansotera, M.; Dardani, P.; Tortelli, V. J. Fluorine Chem. 2012, 140, 43.

[12] (a) Zheng, W. J.; Morales-Rivera, C. A.; Lee, J. W.; Liu, P.; Ngai, M. Y. Angew. Chem., Int. Ed. 2018, 57, 9645.

(b) Zheng, W. J.; Lee, J. W.; Morales-Rivera, C. A.; Liu, P.; Ngai, M. Y. Angew. Chem., Int. Ed. 2018, 57, 13795.

[13] Jelier, B. J.; Tripet, P. F.; Pietrasiak, E.; Franzoni, I.; Jeschke, G.; Togni, A. Angew. Chem., Int. Ed. 2018, 57, 13784.

[14] (a) Noftle, R. E.; Cady, G. H. Inorg. Chem, 1965, 4, 1010.

(b) Katsuhara, Y.; DesMarteau, D. D. J. Am. Chem. Soc. 1980, 102, 2681.

[15] (a) Kolomeitsev, A. A.; Vorobyev, M.; Gillandt, H. Tetrahedron Lett. 2008, 49, 449.

(b) Ignatyev, N.; Hierse, W.; Seidel, M.; Bathe, A.; Schroeter, J.; Koppe, K.; Meier, T.; Barthen, P.; Frank, W. DE 102008024221 , 2009.

(c) Ignatyev, N.; Hierse, W.; Seidel, M.; Bathe, M.; Schroeter, J.; Koppe, K.; Meier, T.; Barthen, P.; Frank, W. WO 2009141053, 2009.

(d) Marrec, O.; Billard, T.; Vors, J. P.; Pazenok, S.; Langlois, B. R. J. Fluorine Chem. 2010, 131, 200.

(e) Sokolenko, T. M.; Davydova, Y. A.; Yagupol'skii, Y. L. J. Fluorine Chem. 2012, 136, 20.

[16] (a) Barbion, J.; Pazenok, S.; Vors, J. P.; Langlois, B. R.; Billard, T. Org. Process Res. Dev. 2014, 18, 1037.

(b) Chen, C. H.; Chen, P. H.; Liu, G. S. J. Am. Chem. Soc. 2015, 137,15648

(c) Chen, C. H.; Luo, Y. X.; Fu, L.; Chen, P. H.; Lan, Y.; Liu, G. S.
J. Am. Chem. Soc. 2018, 140, 1207.

(d) Chen, S.; Huang, Y.; Fang, X.; Li, H.; Zhang, Z.; Hor, T. S. A.; Weng, Z. Dalton Trans. 2015, 44, 19682.

(e) Zha, G. F.; Han, J. B.; Hu, X. Q.; Qin, H. L.; Fang, W. Y.; Zhang, C. P. Chem. Commun. 2016, 52, 7458 .

(f) Chen, D.; Lu, L.; Shen, Q. L. Org. Chem. Front. 2019, 6, 1801.

(g) Yang, Y.; Yao, J.; Yan, W.; Luo, Z.; Tang, Z. Org. Lett. 2019, 21, 8003.

(h) Chen, C. H.; Hou, C. Q.; Chen, P. H.; Liu, G. S. Chin. J. Chem. 2020, $38,346$.

[17] (a) Qi, X. X.; Chen, P. H.; Liu, G. S. Angew. Chem., Int. Ed. 2017, $56,9517$.

(b) Chen, C. H.; Pfluger, P. M.; Chen, P. H.; Liu, G. S. Angew. Chem., Int. Ed. 2019, 58, 2392.

[18] (a) Farnham, W. B.; Smart, B. E.; Middleton, W. J.; Calabrese, J. C.; Dixon, D. A. J. Am. Chem. Soc. 1985, 107, 4565.

(b) Huang, C.; Liang, T.; Harada, S.; Lee, E.; Ritter, T. J. Am. Chem. Soc. 2011, 133, 13308.

[19] Newton, J. J.; Jilier, B. J.; Meanwell, M.; Martin, R. E.; Britton, R.; Friesen, C. M. Org. Lett. 2020, 22, 1785.

[20] Marrec, O.; Billard, T.; Vors, J.; Pazenok, P.; Langlois, B. R. Adv. Synth. Catal. 2010, 352: 2831.

[21] (a) Guo, S.; Cong, F.; Guo, R.; Wang, L.; Tang, P P. Nat. Chem. $\mathbf{2 0 1 7}, 9,546$

(b) Jiang, X. H.; Deng, Z. J.; Tang, P. P. Angew. Chem., Int. Ed. 2018, 130, 298.

(c) Liu, J.; Wei, Y. L.; Tang, P. P. J. Am. Chem. Soc. 2018, 140, 15194.

(d) Cong, F.; Wei, Y. L.; Tang, P. P. Chem. Commun. 2018, 54, 4473.

(e) Yang, H. D.; Wang, F.; Jiang, X. H.; Zhou, Y.; Xu, X. F.; Tang, P. P. Angew. Chem., Int. Ed. 2018, 57. 13266.

(f) Wang, F.; Xu, P.; Cong, F.; Tang, P. P. Chem. Sci. 2018, 9, 8836.

(g) Yang, S. Q.; Chen, M.; Tang, P. P. Angew. Chem., Int. Ed. 2019, 58,7840 .

[22] Zhou, M.; Ni, C.; Zeng, Y.; Hu, J. B. J. Am. Chem. Soc. 2018, 140, 6801.

[23] Li. Y.; Yang, Y.; Xin, J. R.; Tang, P. P. Nat. Commun. 2020, 11, 755.

[24] Xu, P.; Guo, S.; Wang, L. Y.; Tang, P. P. Angew. Chem., Int. Ed. 2014, 53, 5955.

[25] Xu, P.; Wang, F.; Fan, G. L.; Xu, X. F.; Tang, P. P. Angew. Chem., Int. Ed. 2017, 56, 1101. 